\title{
Comparison of two training programmes in chronic airway limitation patients: standardized versus individualized protocols
}

\author{
G. Vallet*, S. Ahmaïdi**, I. Serres**, C. Fabre**, \\ D. Bourgouin*, J. Desplan*, A. Varray+, Ch. Préfaut**
}

Comparison of two training programmes in chronic airway limitation patients: standardized versus individualized protocols. G. Vallet, S. Ahmaïdi, I. Serres, C. Fabre, D. Bourgouin, J. Desplan, A. Varray, Ch. Préfaut. (CERS Journals Ltd 1997.

ABSTRACT: This study tested the effect of two methods of training, one individualized at the heart rate corresponding to the gas exchange threshold (GET) and the other at the heart rate corresponding to $50 \%$ of maximal heart rate reserve, on maximal and submaximal cardiorespiratory response in 24 patients with chronic airway limitation (CAL).

The patients were randomly assigned to either the individualized training group (IT; $\mathbf{n = 1 2 )}$ or the standardized training group (ST; $\mathbf{n = 1 2}$ ). The training programme consisted of 4 weeks of stationary bicycle exercise, 5 days $\cdot$ week $^{-1}$.

Before reconditioning began, the target level based on heart rate was not significantly different between groups $\left(109 \pm 4\right.$ versus $110 \pm 3$ beats $\cdot \mathrm{min}^{-1}$, in IT and ST, respectively). Post-training, a significant increase in symptom-limited oxygen uptake $\left(V^{\prime} \mathrm{O}_{2}, \mathrm{sl}\right)$ and maximal $\mathrm{O}_{2}$ pulse was found in IT, whereas ST exhibited no significant change. In each group, GET was statistically increased in much the same way as $V^{\prime} \mathrm{O}_{2}$,sl, with a higher increase in IT $(\mathrm{p}<0.01)$ than ST $(\mathrm{p}<0.05)$. Nevertheless, IT exhibited a concomitant and gradual decrease in minute ventilation $\left(V^{\prime} \mathrm{E}\right)$, carbon dioxide production $\left(V^{\prime} \mathrm{CO}_{2}\right)$, and venous lactate concentration ([La]), whereas ST presented no significant change in these parameters (intergroup $\mathrm{p}<0.01$ ). Breathing pattern was also altered after IT, at the same metabolic level and at the same ventilation level (intergroup $\mathbf{p}<\mathbf{0 . 0 5}$ ). Cardiac responses were modified in the two groups. At the same metabolic level, a significantly lower cardiac frequency was found both for IT and ST (intragroup $\mathrm{p}<\mathbf{0 . 0 5}$ after training). In contrast, the increase in $\mathrm{O}_{2}$ pulse was only significantly higher in IT after training.

These data show the greater efficiency of an individualized training protocol based on determination of gas exchange threshold as compared to a standardized protocol, in improving exercise performance, when applied to a patient group. Despite an apparently similar target training level, the individualized method clearly optimized the physiological training effects in patients with chronic airway limitation and, more particularly, decreased their ventilatory requirement.

Eur Respir J., 1997; 10: 114-122.
*Clinique du Souffle la Solane, Osséja, France. **Laboratoire de Physiologie des Interactions, Service E.F.R, Hôpital Arnaud de Villeneuve, Montpellier, France. +UFRSTAPS, Laboratoire Sport Santé Développement, Montpellier, France.

Correspondence: Ch. Préfaut

Laboratoire de Physiologie des Interactions Service E.F.R

Hôpital Arnaud de Villeneuve

371 Av Doyen Gaston Giraud

34295 Montpellier Cedex 5

France

Keywords: Chronic airway limitation exercise training

gas exchange threshold

rehabilitation

ventilatory requirement

Received: July 111995

Accepted after revision June 261996

Supported by grant No. 931102 from Institut National de la Santé et de la Recherche Médicale (INSERM).
The intensity of exercise in training programmes for patients with chronic airway limitation (CAL) markedly influences physiological responses $[1,2]$. It is widelyaccepted that an intensity of training exists under which no training effects occur. A recent study by CASABURI et al. [2] showed that high intensity was more efficient than low intensity. In CAL patients, the specificity of the exercise response pattern, characterized by disproportionate ventilation at submaximal levels, is a limiting factor of exercise tolerance. Indeed, the hyperventilation that induces dyspnoea during exercise [3, 4], associated with low aerobic physical fitness due to extensive deconditioning, requires a specific training approach from a ventilatory and metabolic point of view. Despite the substantial training benefits observed in studies of CAL patients $[5,6]$, very few have used the individualized concept. For the last $20 \mathrm{yrs}$, the intensity of exercise in training prescriptions has been based on criteria such as a percentage of maximal oxygen uptake [7] or maximal heart rate [8-11], or an empirical level defined by "as tolerated" or "highest level tolerated" [12, 13], etc. Although the literature reports positive effects from these nonindividualized methods, the problem of optimizing training arises, because a mismatch could potentially exist between the proposed training intensity and the individual exercise ability of the patients.

We validated a method for determining an individualized training intensity based on the heart rate recorded at the gas exchange threshold (GET), described by WASSERMAN et al. [14] as the anaerobic threshold, in asthmatics [15, 16] and CAL patients [17]. This GET could be a better basis for prescription of activity than an arbitrary relative percentage method, for at least three reasons. Firstly, the GET differs between people as a 
function of cardiorespiratory fitness [18], but also of health, age, etc. Secondly, GET does not occur at the same percentage of symptom-limited oxygen uptake $\left(V^{\prime} \mathrm{O}_{2}, \mathrm{sl}\right)$ for all individuals; which means that GET allows precise training prescriptions that take into account an individual and specific metabolic stress. Thirdly, GET represents the breakpoint in ventilation rise; thus, we can expect a good compliance with the training programme by avoiding excessive dyspnoea and/or respiratory disturbance [19]. Based on the above considerations, we can hypothesize that, for the same heart rate used during training, the effects induced will differ according to whether or not they correspond to the GET heart rate.

The aim of this study was, thus, to compare two methods of training, one individualized at the heart rate corresponding to GET, and the other using a standardized formula of $50 \%$ of maximal heart rate reserve [20].

\section{Methods and materials}

\section{Subjects}

Twenty eight subjects with CAL were recruited from an in-patient pulmonary rehabilitation programme at the Clinique du Souffle "la Solane" (Osseja, France). These patients had the following characteristics: 1) dyspnoea while walking ranging 2-3 on the Sadoul scale [21], which corresponds to breathlessness while hill walking at a normal pace and breathlessness while walking at the average pace of a healthy accompanying subject; 2) absence of other disabling diseases; 3) ability to exercise to maximal capacity without adverse cardiovascular effects; 4) minimal bronchodilatator response, ranging $5-20 \%$ of reversibility from baseline (and it should be noted that subjects received the same medical treatment during the training programme, generally limited to theophylline and $\beta_{2}$-agonist agents); 5) lack of regular training activity for more than 2 yrs; and 6) a history of cigarette smoking evaluated at 40 pack-years.
All patients had discontinued smoking prior to entering the programme. They had spirometric evidence of CAL forced expiratory volume in one second (FEV1) $<70 \%$ of predicted, with a reduced $\mathrm{FEV} 1 /$ forced vital capacity (FVC) ratio (table 1), and a clinical history consistent with it. Respiratory impairment ranged from moderate to moderately severe, according to the American Thoracic Society classification [22], and all patients were in a stable phase of their disease. Patients with hospitalization or severe exacerbations 1 month prior to the initiation of the study were excluded from the study. All subjects gave informed consent for participation in this study and were divided at random into two groups of 14 subjects: the first was an individualized training group (IT; $n=14)$; and the second was a standardized training group (ST: $n=14$ ). Four subjects were excluded from the study. Two from the IT group were excluded following episodes of secondary infection, and two from the ST group because of premature departures from the clinic. The results for 24 subjects are presented.

In addition to the training programme, a rehabilitation programme was also offered to all subjects, including education for health and sessions of respiratory therapy. Education for health consisted of twice weekly group sessions on the anatomical and physiological bases of CAL, information on medication and advice on how to manage CAL in everyday life. Respiratory therapy consisted of daily sessions of respiratory retraining and bronchial hygiene, during which the patients were instructed on how to breathe in the most effective way in different situations and how to clean their lungs using simple postures. These sessions were supervised by a specialist. The most important components of respiratory training were pursed-lip and diaphragmatic breathing.

\section{Spirometrics and blood gas measurements}

Maximum expiratory flow-volume (MEFV) curves were recorded on a digital spirometer (Datalink Pulmochart,

Table 1. - Anthropometric characteristics, resting pulmonary function and blood gas values in IT and ST, before and after training

\begin{tabular}{|c|c|c|c|c|}
\hline & \multicolumn{2}{|c|}{ IT $(n=12)$} & \multicolumn{2}{|c|}{$\mathrm{ST}(\mathrm{n}=12)$} \\
\hline & Pretraining & Post-training & Pretraining & Post-training \\
\hline Age yrs & $55 \pm 3$ & & $59 \pm 3$ & \\
\hline Body mass kg & $74 \pm 4$ & $74 \pm 4$ & $69 \pm 2$ & $69 \pm 2$ \\
\hline Height $\mathrm{cm}$ & $171 \pm 3$ & & $166 \pm 2$ & \\
\hline FVC L & $3.0 \pm 0.3$ & $3.3 \pm 0.3$ & $3.3 \pm 0.2$ & $3.3 \pm 0.3$ \\
\hline$\%$ pred & (77) & $(85)$ & (93) & (91) \\
\hline $\mathrm{FEV}_{1} \mathrm{~L} \cdot \mathrm{s}^{-1}$ & $1.73 \pm 0.18$ & $1.77 \pm 0.18$ & $1.82 \pm 0.15$ & $1.89 \pm 0.17$ \\
\hline$\%$ pred & $(54)$ & $(57)$ & (63) & (66) \\
\hline $\mathrm{FEV}_{1} / \mathrm{FVC} \%$ & $57 \pm 3$ & $55 \pm 3$ & $55 \pm 3$ & $57 \pm 3$ \\
\hline $\mathrm{Pa}_{\mathrm{a}, \mathrm{O}_{2}} \quad \mathrm{kPa}$ & $9.4 \pm 0.3$ & $9.6 \pm 0.3$ & $9.4 \pm 0.3$ & $9.5 \pm 0.2$ \\
\hline $\mathrm{mmHg}$ & $70.2 \pm 2.2$ & $71.9 \pm 2.0$ & $70.8 \pm 2.0$ & $71.3 \pm 1.8$ \\
\hline$P_{\mathrm{a}, \mathrm{CO}_{2}} \quad \mathrm{kPa}$ & $4.7 \pm 0.2$ & $4.6 \pm 0.2$ & $4.8 \pm 0.1$ & $4.8 \pm 0.1$ \\
\hline $\mathrm{mmHg}$ & $35.3 \pm 1.3$ & $34.6 \pm 1.3$ & $36.0 \pm 1.0$ & $35.9 \pm 0.8$ \\
\hline $\mathrm{pH}$ & $7.42 \pm 0.01$ & $7.43 \pm 0.01$ & $7.42 \pm 0.01$ & $7.42 \pm 0.01$ \\
\hline Sex ratio $\mathrm{M} / \mathrm{F}$ & $8 / 4$ & & $10 / 2$ & \\
\hline
\end{tabular}

All values are means \pm SEM. Values in parenthesis are $\%$ of theoretical value. IT: individualized training; ST: standardized training; F: female; M: male; n: number of subjects; FEV1: forced expiratory volume in one second; FVC: forced vital capacity; $P$ a, $\mathrm{O}_{2}$ : arterial oxygen tension; $\mathrm{Pa}_{\mathrm{a}} \mathrm{CO}_{2}$ : arterial carbon dioxide tension. There was no significant intra- or intergroup difference before and after training. 
Montpellier, France). The lung function study included FVC, FEV1, maximal expiratory flow at $50 \%$ of FVC (MEF50), and forced expiratory flow between 25 and $75 \%$ of FVC (FEF25-75\%). The values of FEV1/FVC were then calculated. The predicted values were those of QuANJER [23].

Arterial blood pressure was measured from an artery of the nondominant arm at $37^{\circ} \mathrm{C}$ using a gas analyser (IL2 meter, Milan, Italy).

\section{Exercise testing}

An incremental exercise test was performed on a calibrated cycle ergometer (Ergolyne Ergometrics 900, Germany) according to the individualized exercise test protocol used in our laboratory, and defined as the following: first, the maximal predicted work load was calculated and adapted to the patient by multiplication of the $\%$ fall in FEV1 by comparison to the predicted value. The 3 min warm-up was conducted at $20 \%$ of this maximal estimated workload. The workload was then increased every minute. The increase rate was defined as $8 \%$ of maximal estimated workload, in order to obtain the maximal workload in about $10 \mathrm{~min}$.

Oxygen uptake $\left(V^{\prime} \mathrm{O}_{2}\right), \mathrm{CO}_{2}$ output $\left(V^{\prime} \mathrm{CO}_{2}\right)$ and ventilation $\left(V^{\prime} \mathrm{E}\right)$, with its components tidal volume $(V \mathrm{~T})$, respiratory frequency $\left(f^{\prime} R\right)$, and respiratory exchange ratio (R), were recorded continuously using a breath-by-breath automated exercise metabolic system but measured on the last $20 \mathrm{~s}$ of each load over an integral number of cycles (CPX; Medical Graphics, St Paul, MN, USA). Expiratory airflow was measured with a pneumotachograph (Type 3, 3800; Hans Rudolph Inc., Kansas City, KS, USA) connected to a pressure transducer (DP 25014; Validyne Engineering Corp., CA, USA). Expired gases were analysed for $\mathrm{O}_{2}$ with zirconia solid electrolyte $\mathrm{O}_{2}$ analysers, and for $\mathrm{CO}_{2}$ with an infra-red analyser. Before each test, the volume was calibrated by five inspiratory and expiratory strokes with a $3 \mathrm{~L}$ pump; the gas analyser, with two mixtures of gases of known oxygen and carbon dioxide concentration. To ensure that maximal oxygen uptake $\left(V^{\prime} \mathrm{O}_{2}\right.$, max $)$ was attained, at least three of the following criteria had to be met: 1) an increase in $V^{\prime} \mathrm{O}_{2}<5 \mathrm{~mL}$ with the last increase in work rate; 2 ) attainment of age-predicted maximal cardiac frequency $(210-0.65$ age $\pm 10 \%) ; 3$ ) a respiratory exchange ratio $>1.10$; and 4 ) an inability to maintain the required pedalling frequency $\left(60 \mathrm{rev} \cdot \mathrm{min}^{-1}\right)$ despite maximal effort and verbal encouragement. If one of the three points was not observed, we considered that the $V^{\prime} \mathrm{O}_{2}$, max was $V^{\prime} \mathrm{O}_{2}$ symptom-limited $\left(V^{\prime} \mathrm{O}_{2}\right.$, sl $)$. A 3-lead electrocardiograph (ECG) (DII, V2; V5) was monitored continuously during the exercise tests. The blood pressure was continuously recorded on a tensiometer (Quinton Q 3000; Seattle, WA, USA). The oxygen pulse was computed from $V^{\prime} \mathrm{O}_{2}$ and $f^{\prime} \mathrm{R}$, and expressed in $\mathrm{mL} \cdot \mathrm{kg}^{-1}$. beat ${ }^{-1}$.

\section{Determination of gas exchange threshold (GET)}

Cardiorespiratory variables used to define GET were determined continuously by the moving average of eight respiratory cycles. After completion of the exercise test, the GET was determined for each subject by using the modified V-slope method described by SuE et al. [24].
This method involves the analysis of the behaviour of $V^{\prime} \mathrm{CO}_{2}$ as a function of $V^{\prime} \mathrm{O}_{2}$, and assumes that GET corresponds to the breakpoint in the $V^{\prime} \mathrm{CO}_{2}-V^{\prime} \mathrm{O}_{2}$ relationship. The identification of the GET was made blindly and independently by two experienced reviewers from coded photocopies of the data. The reviewers had no access to names, groups or test dates. If the GET determinations were not exactly the same for the two reviewers, but corresponded closely at a similar cardiac frequency $\left(f^{\prime} \mathrm{C}\right)( \pm 5$ beats), the average of the two independent determinations was taken.

\section{Blood lactate analyses}

A blood sample was drawn from an antecubital vein in the nondominant arm whilst at rest and during the last $10 \mathrm{~s}$ of each minute during testing. Blood was immediately placed in a tube containing ethylenediaminetetra-acetic acid (EDTA) and kept in ice. Plasma was separated within $15 \mathrm{~min}$ after blood collection by using a refrigerated centrifuge. Plasma was then frozen $\left(-80^{\circ} \mathrm{C}\right)$ until assayed. Blood lactate analyses were made using an enzymatic method (Boehringher kit, Mannheim, FRG) with a centrifuge analyser (Cobas Bio, Roche, France). For technical reasons, lactate concentration ([La]) was measured in only seven patients in each group.

\section{Training programme}

Patients were instructed to perform one session of stationary bicycle exercise per day, 5 days per week, over a 4 week period. The target level was based on the cardiac frequency, either corresponding to GET or at the standard level of $50 \%$ of maximal cardiac frequency reserve. The training schedule was the same in the two groups, consisting of a 5 min warm-up followed by 10 min of work and $5 \mathrm{~min}$ of active recovery, repeated over a 45 min session. During training, $f^{\prime} \mathrm{C}$ was continuously monitored by means of a cardiofrequency meter (Sport Tester PE 3000; Polar Electro, Kemple, Finland). The cardiofrequency meter was set in such a way that subjects could exercise within \pm 5 beats $\cdot \mathrm{min}^{-1}$ of prescribed intensity. An alarm ensured that the subject trained within the preselected range. To verify that training procedures were followed, an instructor supervised each session, without knowing to which group the patients belonged. No instructions were given concerning breathing pattern.

\section{Rehabilitation programme}

For both groups, the additional components of rehabilitation were exactly the same. For example, respiratory therapy was carried out by the same therapist using the same methods for all patients.

\section{Protocol}

The subjects were tested pretraining (pre) and after the 4 week training period (post). After the first test, the subjects were randomized into two groups until a comparable mean training cardiac frequency was obtained for the two groups (table 2). ST was trained at the 
Table 2. - Values of cardiac frequency used for intensity training in the two groups

\begin{tabular}{|c|c|c|c|}
\hline \multirow{2}{*}{$\begin{array}{l}\text { Ss } \\
\text { No. }\end{array}$} & \multicolumn{3}{|c|}{ Cardiac frequency beats $\cdot \mathrm{min}^{-1}$} \\
\hline & $f^{\prime} \mathrm{C}, \max$ & $f^{\prime}$ C,rest & Training ${ }^{+}$ \\
\hline \multicolumn{4}{|c|}{ Individualized training } \\
\hline 1 & 150 & 90 & 119 \\
\hline 2 & 120 & 54 & 99 \\
\hline 3 & 144 & 70 & 113 \\
\hline 4 & 109 & 68 & 90 \\
\hline 5 & 147 & 79 & 127 \\
\hline 6 & 115 & 57 & 87 \\
\hline 7 & 169 & 83 & 112 \\
\hline 8 & 133 & 65 & 107 \\
\hline 9 & 169 & 100 & 137 \\
\hline 10 & 152 & 59 & 121 \\
\hline 11 & 115 & 84 & 103 \\
\hline 12 & 114 & 76 & 95 \\
\hline Mean & 136 & 74 & 109 \\
\hline SEM & 6 & 4 & 4 \\
\hline \multicolumn{4}{|c|}{ Standardized training } \\
\hline 1 & 137 & 79 & 108 \\
\hline 2 & 135 & 69 & 102 \\
\hline 3 & 168 & 92 & 130 \\
\hline 4 & 147 & 61 & 104 \\
\hline 5 & 147 & 69 & 108 \\
\hline 6 & 138 & 102 & 120 \\
\hline 7 & 105 & 77 & 91 \\
\hline 8 & 141 & 65 & 103 \\
\hline 9 & 146 & 68 & 107 \\
\hline 10 & 131 & 73 & 102 \\
\hline 11 & 182 & 70 & 126 \\
\hline 12 & 154 & 90 & 122 \\
\hline Mean & 144 & 76 & 110 \\
\hline SEM & 6 & 4 & 3 \\
\hline \multirow[t]{2}{*}{ p-value } & 0.50 & 0.90 & 0.90 \\
\hline & NS & NS & NS \\
\hline
\end{tabular}

$+:$ the cardiac frequency used in the training programmes were $f^{\prime} \mathrm{C}, \mathrm{GET}$ in the individualized training group and $50 \% \mathrm{f}^{\prime} \mathrm{C}, \mathrm{r}$ in the standardized training group. $f^{\prime} \mathrm{C}$,max: maximal cardiac frequency; $f^{\prime} \mathrm{C}$,rest: cardiac frequency; $f^{\prime} \mathrm{C}, \mathrm{GET}$ : cardiac frequency at gas exchange threshold; $50 \% \mathrm{f}^{\prime} \mathrm{C}, \mathrm{r}$ : cardiac frequency corresponding to $50 \%$ of maximal cardiac frequency; Ss: subjects; Ns: nonsignificant intergroup difference.

standard level of $50 \%$ maximal cardiac frequency reserve $\left(50 \% f^{\prime} \mathrm{C}, \mathrm{r}\right)$ calculated from the formula given by the American College of Sports Medicine [20]:

$$
\left(50 \%\left(f^{\prime} \mathrm{C}, \max -f^{\prime} \mathrm{C}, \text { rest }\right)+f^{\prime} \mathrm{C}, \text { rest }\right)
$$

Resting cardiac frequency $\left(f^{\prime} \mathrm{C}\right.$,rest) was determined in the morning, and maximal cardiac frequency $\left(f^{\prime} \mathrm{C}, \max \right)$ at maximal exercise during the first maximal exercise test. For the elderly, it is recommended that beginners with low initial fitness be trained at $50-75 \%$ of maximal reserve [20, 25]. The intensity used in the ST group was $50 \%$ maximal $f^{\prime} \mathrm{C}$, r to take account of age and low initial fitness.

In contrast, the IT group was trained at an intensity corresponding to the GET cardiac frequency $\left(f^{\prime} \mathrm{C}, \mathrm{GET}\right)$. As shown in table 2, the cardiac frequencies used for training in each group were not statistically different. The subjects and the exercise test supervisor did not know which method had been used to determine the training cardiac frequency.

\section{Statistical analysis}

The values are reported as mean \pm SEM. The anthropometric, spirometric and training cardiac frequency data collected on entry were compared between the two groups with an unpaired Student's t-test.

In ST, the agreement between the $f^{\prime}$ C,GET and 50\% $f^{\prime} \mathrm{C}, \mathrm{r}$ was assessed using the method of BLAND and ALTMAN [26], which plots the $f^{\prime} \mathrm{C}, \mathrm{GET}-50 \% f^{\prime} \mathrm{C}, \mathrm{r}$ differences against the means of these two cardiac frequencies.

Maximal exercise and spirometric values, and preand post-training data, were analysed for statistical significance by using a two-way analysis of variance (ANOVA) (group $\times$ time). When the ANOVA $F$ ratio was significant $(\mathrm{p}<0.05)$, the means were compared by using the post-hoc contrast method.

Analysis at submaximal level was carried out as follows:

1. $V^{\prime} \mathrm{O}_{2}$ was plotted against $V^{\prime} \mathrm{E}, f^{\prime} \mathrm{C}, \mathrm{O}_{2}$ pulse, $V \mathrm{~T}, f^{\prime} \mathrm{R}$, and [La], and $V^{\prime} \mathrm{E}$ against $f^{\prime} \mathrm{R}$, and $V \mathrm{~T}$, for each subject. A line of best fit for each relationship was determined for each plot (among polynomial, linear, power, logarithmic and exponential).

2. From the line of best fit values, $V^{\prime} \mathrm{E}, f^{\prime} \mathrm{C}, \mathrm{O}_{2}$ pulse, $V \mathrm{~T}, f^{\prime} \mathrm{R}$, and [La] were calculated at five submaximal and maximal workloads, representing 50, 60, 70, 80, 90 and $100 \%$ of the pretraining $V^{\prime} \mathrm{O}_{2}$,sl or $V^{\prime} \mathrm{E}$,max. ST and IT were compared in terms of the values before and after training by using a three-way ANOVA (group $\times$ time $\times$ intensity).

The percentage differences used in table 3 were calculated according to the following formula:

pretraining mean value - posttraining mean value pretraining mean value

Table 3. - Effects of training on maximal cardiorespiratory variables and change in GET

\begin{tabular}{|c|c|c|c|c|c|}
\hline Parameters & Groups & Pretraining & Posttraining & $\%$ Change & p-value \\
\hline \multirow{2}{*}{$V^{\prime} \mathrm{O}_{2}, \mathrm{sl} \quad \mathrm{mL} \cdot \mathrm{kg}^{-1} \cdot \mathrm{min}^{-1}$} & IT & $22.4 \pm 1.1$ & $26.9 \pm 1.3$ & 20 & $<0.05$ \\
\hline & ST & $22.8 \pm 1.2$ & $25.1 \pm 1.3$ & 10 & NS \\
\hline \multirow{2}{*}{ GET $\mathrm{mL} \cdot \mathrm{kg}^{-1} \cdot \mathrm{min}^{-1}$} & IT & $14.4 \pm 0.6$ & $17.6 \pm 0.7$ & 22 & $<0.01$ \\
\hline & ST & $14.5 \pm 0.7$ & $15.7 \pm 0.6$ & 8 & $<0.05$ \\
\hline \multirow{2}{*}{$V^{\prime}$ E,max $\mathrm{L} \cdot \mathrm{min}^{-1}$} & IT & $69.1 \pm 3.2$ & $76.2 \pm 4.8$ & 10 & NS \\
\hline & ST & $63.6 \pm 4.3$ & $68.8 \pm 3.6$ & 8 & NS \\
\hline \multirow[t]{2}{*}{$f^{\prime} \mathrm{C}, \max$ beats $\cdot \mathrm{min}^{-1}$} & IT & $136.4 \pm 5.8$ & $139.3 \pm 5.1$ & 0.6 & NS \\
\hline & ST & $144.2 \pm 5.5$ & $142.3 \pm 5.6$ & -0.5 & NS \\
\hline \multirow{2}{*}{ Maximum $\mathrm{O}_{2}$ pulse $\mathrm{mL} \cdot \mathrm{kg}^{-1} \cdot$ beat $^{-1}$} & IT & $0.12 \pm 0.06$ & $0.14 \pm 0.04$ & 17 & $<0.05$ \\
\hline & ST & $0.11 \pm 0.05$ & $0.12 \pm 0.05$ & 9 & NS \\
\hline
\end{tabular}

Data are presented as mean \pm SEM. GET: gas exchange threshold; $V^{\prime} \mathrm{O}_{2}$,max: maximal oxygen uptake; $V^{\prime} \mathrm{E}$,max: maximal minute ventilation. For further definitions see legends to tables 1 and 2. 
Delta $(\Delta)$ values were calculated by the difference between pre- and post-training values obtained at different percentages of pretraining $V^{\prime} \mathrm{O}_{2}$,sl. The limit for statistical significance was always set at a value less than 0.05 .

\section{Results}

\section{Intensity level and comparison between methods}

Anthropometric characteristics are listed in table 1. There were no differences between the two groups. Before the reconditioning programme, the level of training intensity expressed by mean cardiac frequency was not

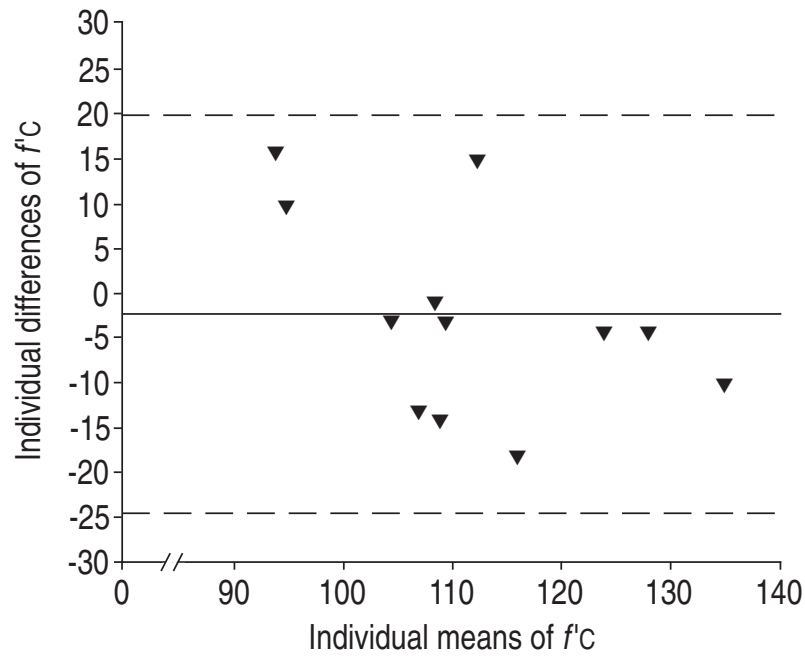

Fig. 1. - Distribution in standardized training (ST) group of differences in cardiac frequency $\left(f^{\prime} \mathrm{C}\right) f^{\prime} \mathrm{C}$, GET and $50 \% f^{\prime} \mathrm{C}, \mathrm{r}$ against the means of these two cardiac frequencies. Intensity stimulation was very different between patients. The horizontal lines indicate mean $\pm 2 \mathrm{sD}$. $f^{\prime} \mathrm{C}, \mathrm{GET}$ : cardiac frequency at gas exchange threshold; $50 \% \mathrm{f}^{\prime} \mathrm{C}$,r: cardiac frequency corresponding to $50 \%$ maximal cardiac frequency reserve. significantly different in each group (table 2). Nevertheless, in ST there was no agreement between $50 \% f^{\prime} \mathrm{C}$,r and $f^{\prime} \mathrm{C}$,GET (fig. 1). The differences between these values of cardiac frequency ranged from 17 beats $\cdot \mathrm{min}^{-1}$ above to 19 beats. mean ${ }^{-1}$ below the GET cardiac frequency. Thus, despite the same target cardiac frequency, most of the ST patients were not trained at the GET intensity but above or below it.

Twenty four patients completed the entire programme, which was well-tolerated, regardless of the training intensity chosen.

\section{Resting values}

The results of mean baseline pulmonary function tests and blood gas measurements in the two groups are listed in table 1 . There were no significant changes in resting pulmonary function and blood gas values before and after training in either group.

\section{Responses to maximal exercise}

As shown in table $3, V^{\prime} \mathrm{O}_{2}$, sl and maximal $\mathrm{O}_{2}$ pulse increased in IT after training $(\mathrm{p}<0.05)$, whereas this improvement did not reach significance in ST. The $V^{\prime} \mathrm{E}$,max and $f^{\prime} \mathrm{C}$,max were not statistically changed, whatever the training method.

\section{Effect of training on submaximal values}

In both groups, the GET was significantly improved (table 3). For a given pretraining $V^{\prime} \mathrm{O}_{2}$, there was a significant difference between the two groups. In the IT group, a gradual and parallel significant decrease was obtained in [La], $V^{\prime} \mathrm{E}$, and $V^{\prime} \mathrm{CO}_{2}$, (fig. 2). In contrast, no greater change was observed in ST concerning these variables.

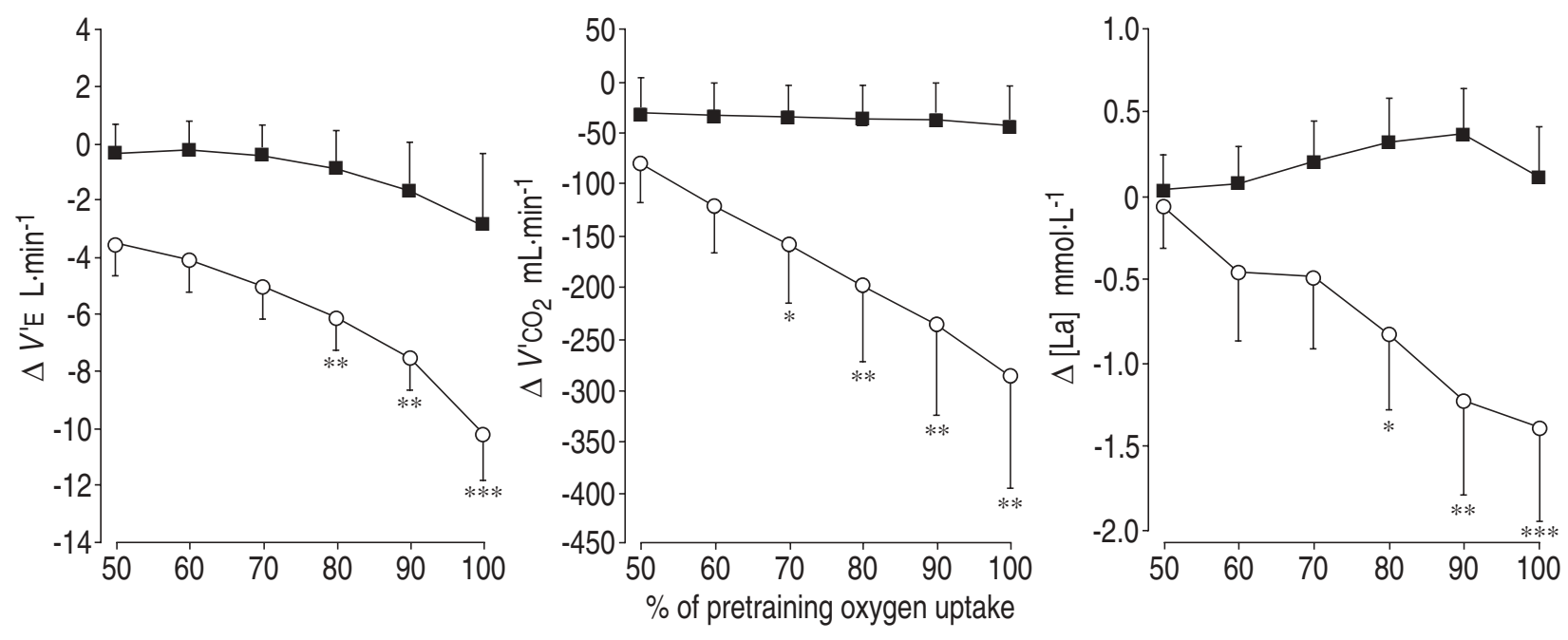

Fig. 2. - Effect of training in IT (- - - ) and ST (- - ) on delta time course of $V^{\prime} \mathrm{E}, V^{\prime} \mathrm{CO}_{2}$ and [La] at different levels of pretraining $V$ ' ${ }_{2}$, sl. Values are presented as delta average \pm SEM. Results are presented for 12 subjects for $V^{\prime} \mathrm{E}$ and $V^{\prime} \mathrm{CO}_{2}$ and for seven subjects for [La]. IT: individualized training; ST: standardized training; $V^{\prime} \mathrm{E}$ : minute ventilation; $V^{\prime} \mathrm{CO}_{2}$ : carbon dioxide output; [La]: blood lactate concentration; $V$ 'O ${ }_{2}$, sl: symptom-limited oxygen uptake. Intergroup comparisons were significant $(\mathrm{p}<0.001)$ in all three cases. $*: \mathrm{p}<0.05 ; * *$ : $\mathrm{p}<0.01 ; * * *: \mathrm{p}<0.001$, significance of intragroup comparison. 

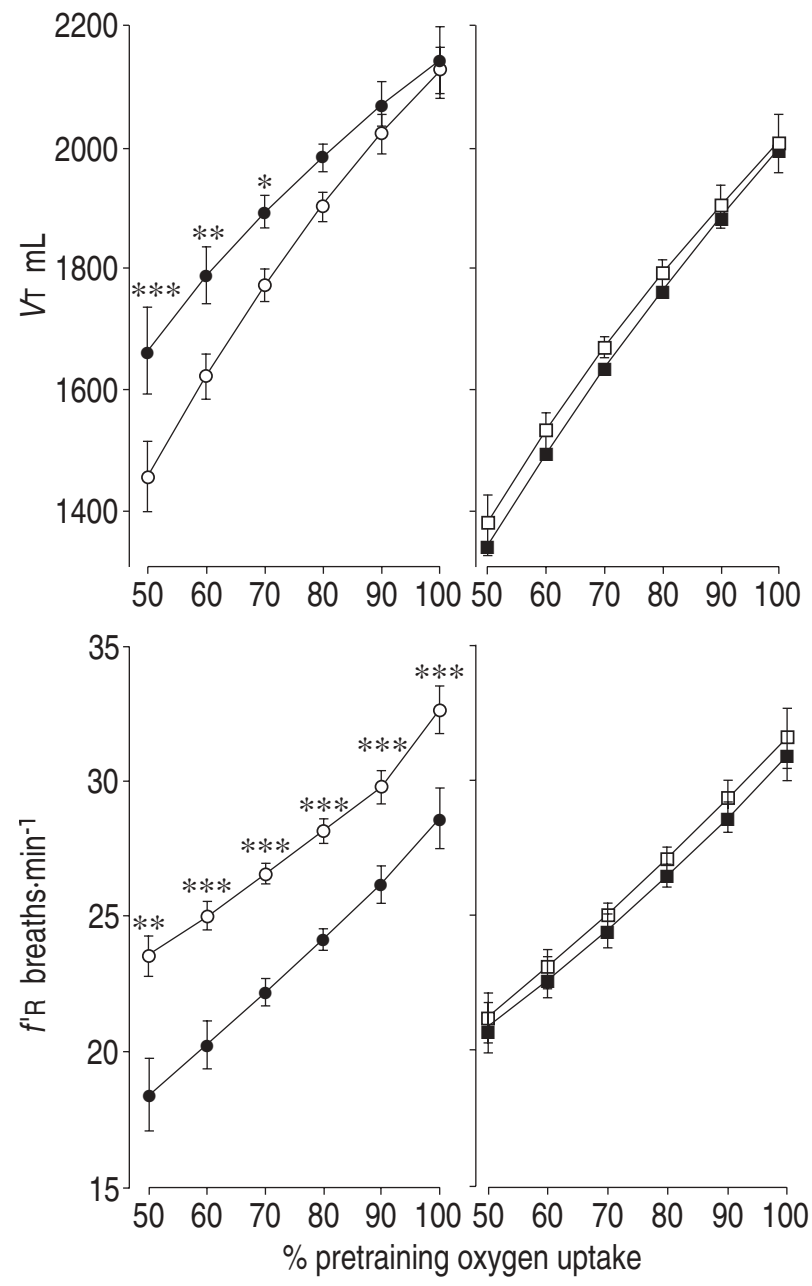

Fig. 3. - Comparison of pre- $(\circ, \square)$ and post-training $(\bullet, \nabla)$ respiratory and tidal volume $(V \mathrm{~T})$ at the same metabolic level, in individualized training (IT) $(\bullet, 0)$ and standardized training (ST) $(\square, \square)$. Values are presented as mean \pm SEM. $*: \mathrm{p}<0.05$; **: $\mathrm{p}<0.01$; $* * *$ : $\mathrm{p}<0.001$, significance of intragroup comparisons of pre- and posttreatment values.

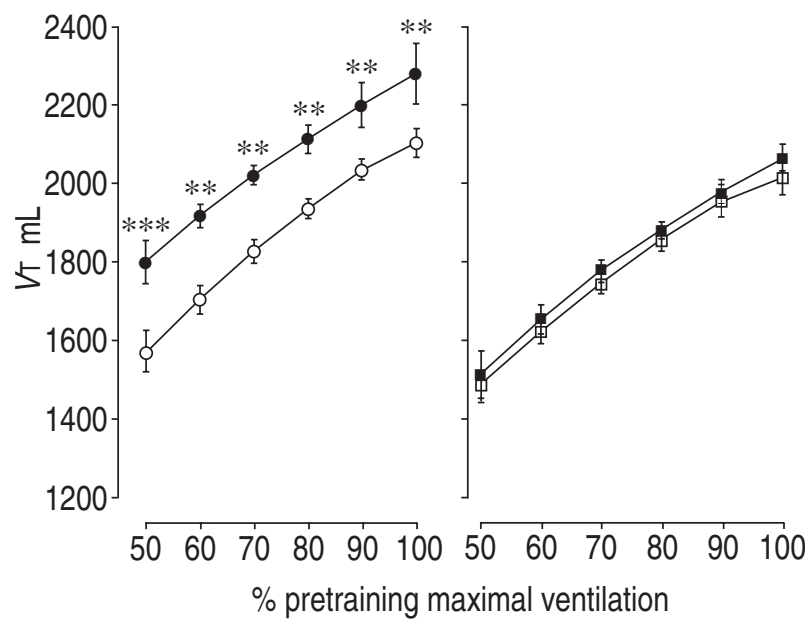

Fig. 4. - Comparison of pre- $(O, \square)$ and post-training $(\bullet, \mathbf{\bullet})$ tidal volume $(V \mathrm{~T})$ at the same ventilation level, in individualized training (IT) $(\bullet, O)$ and the standardized training (ST) $(\square, \square)$. Values are presented as mean \pm SEM. $*$ : $\mathrm{p}<0.05$; **: $\mathrm{p}<0.01$; $* * *: \mathrm{p}<0.001$, significance of intragroup comparisons of pre- and post-treatment values.

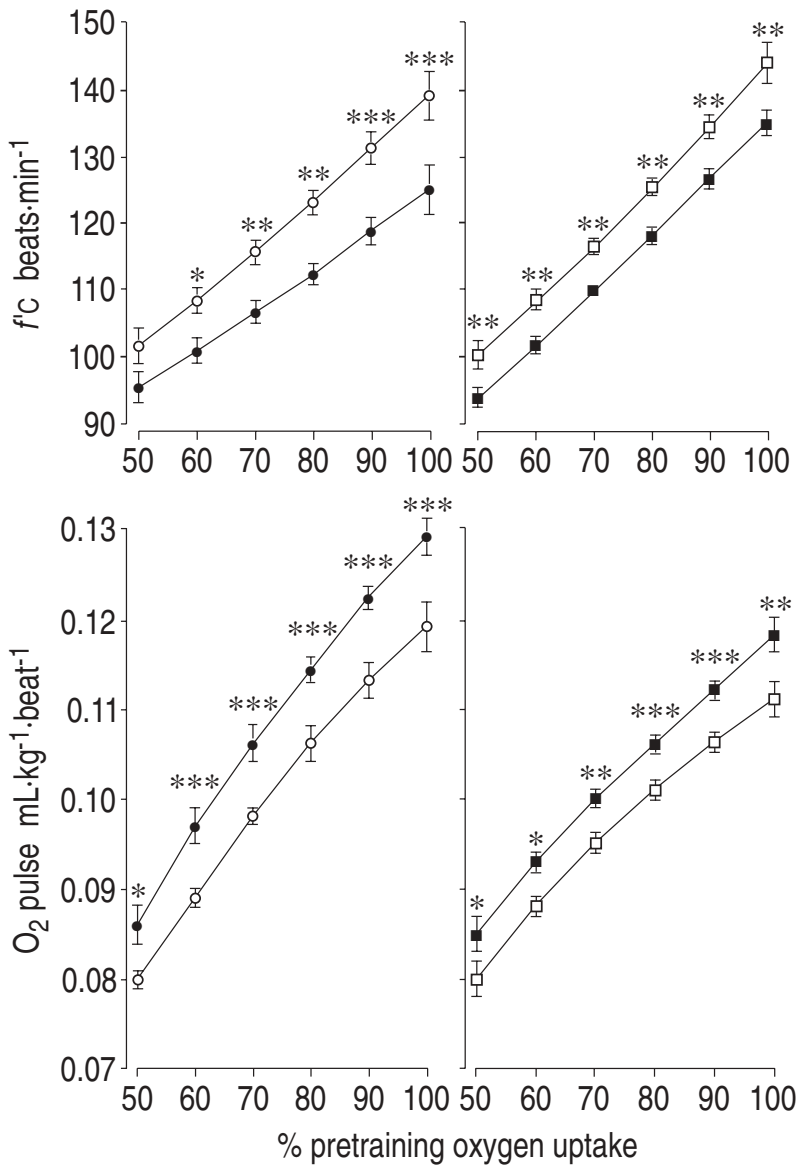

Fig. 5. - Comparison of pre- $(O, \square)$ and post-training $(\bullet, \mathbf{\bullet})$ cardiac frequency and $\mathrm{O}_{2}$ pulse at the same metabolic level, in individualized training (IT) $(\bullet, \bigcirc)$ and standardized training (ST) (घ, $\square)$. Values are presented as mean \pm SEM. $*: \mathrm{p}<0.05 ; * *: \mathrm{p}<0.01 ; * * *: \mathrm{p}<0.001$, significance of intragroup comparison of pre- and post-treatment values.

Breathing pattern was significantly different after training between the two groups $(\mathrm{p}<0.05)$ at the same metabolic level (fig. 3) and for a given level of ventilation (fig. 4). In ST, whatever the metabolic or ventilation level, no significant change was found. In contrast, IT modified the breathing pattern. At the same metabolic level, IT exhibited a systematically lower respiratory frequency and higher tidal volume, especially at 50, 60 and $70 \%$ of pretraining $V^{\prime} \mathrm{O}_{2}$ (fig. 3). For a given level of ventilation (fig. 4), tidal volume was systematically higher after IT at GET. After training, at the same level of pretraining $V^{\prime} \mathrm{O}_{2}$, there was a significantly decreased cardiac frequency and increased $\mathrm{O}_{2}$ pulse both in IT and ST (fig. 5).

\section{Discussion}

The major finding of this study was that, for comparable target cardiac frequencies, IT at GET induced greater physiological effects, i.e. better submaximal cardiorespiratory responses, and a reduction in ventilation and lactic acidosis.

A relative consensus exists on the duration and frequency of training in CAL reconditioning programmes, but there is considerable variability among exercise 
regimens in terms of intensity of training which explains, in part, why programmes of pulmonary rehabilitation yield widely differing physiological training responses $[2,17,27]$. Intensity level is defined by standard criteria that do not necessarily allow the individual patient a comparable stimulus in terms of metabolic stress, because of the heterogeneity in patient physical fitness. Programmes using prescriptions based on the relative percentage concept may create variable training stimuli among the participants, which will in turn result in a wide range of improvement in cardiorespiratory and metabolic function $[1,7,8,25]$. In this study the $50 \%$ maximal heart rate reserve level used to define training intensity in ST did not result in a systematic improvement in cardiorespiratory and metabolic functions in all patients, as compared to IT. The Bland and Altman [26] test showed the great disparity in ST training intensity by comparison with the individualized approach, which was more successful even though we thought that direct application of this principle in training patients with CAL might be prevented by ventilatory limitation linked to the severity of the disease [28].

The GET, described as the anaerobic threshold by WASSERMAN et al. [14], allows the determination of a level of training intensity before a sharp increase in ventilation occurs, and is linked to cardiorespiratory fitness $[18,29]$. This hyperventilation has been interpreted as a consequence of excessive lacticacidaemia [2]. For most CAL patients, who are limited by ventilation capacity, an exercise at an intensity above the ventilatory threshold could induce the following: firstly, the lacticacidaemia will increase and then worsen hyperventilation, thus inducing high respiratory discomfort, dyspnoea [3, 4], and higher oxygen cost for ventilation [30]; secondly, the oxygen uptake needed for lactate removal will be increased, and the combination of the above will decrease the amount of oxygen uptake for exercising muscles. Our hypothesis was that in patients with CAL, GET would determine an effective exercise intensity without the above problems and would be more effective than a standardized approach, which cannot take into account the level of the patient's physical fitness.

\section{Clinical benefits and pulmonary function}

This study failed to demonstrate significant modification of pulmonary function and arterial blood gas values after a training programme with either training strategy. This result is consistent with other studies [8-10].

\section{Response at maximal exercise}

After training there was a more marked increase in $V^{\prime} \mathrm{O}_{2}$,sl in IT, which is consistent with other studies [7, 31, 32]. This difference between the two groups could be explained, firstly, by the high dispersion of training levels in ST compared to IT (fig. 1). In addition, the $\mathrm{O}_{2}$ pulse was significantly increased after training in IT, indicating that the $V^{\prime} \mathrm{O}_{2}$, sl improvement was achieved either by increased peripheral $\mathrm{O}_{2}$ uptake, stroke volume or both.

\section{Training effects}

After the training programme, despite a nonsignificant difference between the two groups, the increase in GET was higher in IT than in ST (22 vs 8\%) (table 3). This result is consistent with other studies conducted in CAL patients [17], young asthmatic subjects [15, 16] and elderly people $[25,31]$ after individualized training programmes. The low GET level found before training in our CAL patients reflects considerable deconditioning and a low aerobic capacity [18, 19]. This result is consistent with previous studies [33]. The discrepancy between the two groups in the level of increase of GET following the training programme could be explained by the randomness inherent in the $50 \% f^{\prime} \mathrm{C}, \mathrm{r}$ formula to define individual training intensity.

After IT, we observed a concomitant and proportional decrease in ventilation $V^{\prime} \mathrm{CO}_{2}$, and a decrease in blood [La] for a given $V^{\prime} \mathrm{O}_{2}$. These results support previous studies in which training was used to reduce ventilation by reducing lactic acidosis [2, 34, 35]. Two previous studies in CAL patients used comparable variables to define training level $[2,36]$. CoRriveau et al. [36] used GET. They obtained less satisfactory results, than those of the present study, probably because of methodological differences in the protocols of the training programmes and laboratory testing. The discrepancy in the use of the one mode of exercise during testing (treadmill) and another during training (stationary bicycle) certainly limited the stimulation of target cardiac frequency and training effects. Secondly, the present results are consistent with the study by CASABURI et al. [2], in which the relationship between the decrease in $V$ 'E and blood [La] was found after a high-intensity (above GET) training programme. In the present study, the intensity used in IT corresponded to an intermediate level between the "low level" (in which no significant change occurred after training) and the "high level" (with significant training effects) used by CASABURI et al. [2].

As shown previously [37], one of the major characteristics of the respiratory pattern during exercise in CAL is "rapid shallow breathing". In the present study, we obtained a positive change in ventilatory pattern after training in IT both for a given metabolic rate and a given ventilation level, whereas ST showed no modification. In IT, at the same metabolic rate, the tidal volume was increased and respiratory frequency was decreased. In addition, at the same ventilation level, tidal volume was systematically higher, and respiratory rate was lower. This new respiratory strategy is very interesting for two reasons: firstly, a higher tidal volume for a given $V^{\prime} E$ may result in a lower dead space ventilation, which leads to a more effective alveolar ventilation [38]; and, secondly, the lower respiratory frequency found after training is also a very good response to exercise, because it supports a decreased sensation of dyspnoea [39]. Despite a slight improvement in GET, no change in respiratory pattern was observed in ST.

An effect of hyperventilation on cardiovascular function has been described $[40,41]$ and this has also been suggested as an explanation of the reduced aerobic capacity in CAL patients $[8,9]$. In the present study, better cardiac adaptation at submaximal level was obtained in IT, while reduction in hyperventilation was also more 
marked. Whether or not there is a link between these physiological changes, the individualized training protocol is more "efficient" and sensitive.

In summary, the major finding of this study was that despite an apparently identical training intensity expressed by the same mean level of cardiac frequency, an individualized training method and a standardized training method did not induce the same magnitude of physiological effects. While standardized training is convenient when applied to groups of patients, and can be demonstrated to show overall benefit, individualized training is more likely to produce optimal benefit for each patient, a key strategy in pulmonary rehabilitation.

Acknowledgements: The authors wish to thank J. Martrés, M. Guerzou, F. Ceugniet, J. Cabezudo, S. Echard and C. Deysquien for their technical assistance and generous contribution of time. They also wish to thank the subjects for their co-operative attitude and dedicated performance.

\section{References}

1. Punzal PA, Ries Al, Kaplan RM, Prewitt LM. Maximum intensity exercise training in patients with chronic obstructive pulmonary disease. Chest 1991; 100: 618-623.

2. Casaburi R, Patessio A, Ioli F, Zanaboni S, Donner C, Wasserman K. Reductions in exercise lactic acidosis and ventilation as a result of exercise training in patients with obstructive lung disease. Am Rev Respir Dis 1991; 143: 9-18.

3. Lachman A, Sanna A, De Coster J, Sergysels R. Evaluation of dyspnea during exercise in COPD patients. Am Rev Respir Dis 1990; A830.

4. Sanna A, Lachman A, Sergysels R. Dyspnea during exercise in normal subjects. Eur Respir Rev 1989; 2 (Suppl. 5): 385s.

5. Goldstein RS, Gort EH, Stubbing D, Avendano MA Guyatt Gh. Randomised controlled trial of respiratory rehabilitation. Lancet 1994; 344: 1394-1397.

6. Toshima MT, Kaplan RM, Ries AL. Experimental evaluation of rehabilitation in chronic obstructive pulmonary disease: short-term effects on exercise endurance and health status. Health Psychol 1990; 9: 237-252.

7. Holle R, Williams VD, Vandree JC, Starcks GL, Schoene RB. Increased muscle efficiency and sustained benefits in an out-patient community hospital-based pulmonary rehabilitation program. Chest 1988; 94: 1161-1168.

8. Degre S, Sergysels R, Messin R, et al. Hemodynamic responses to physical training in patients with chronic lung disease. Am Rev Respir Dis 1974; 110: 395-401.

9. Alpert JS, Bass H, Szucs MM, Banas JS, Dalen JE, Dexter L. Effects of physical training on hemodynamics and pulmonary function at rest and during exercise in patients with COPD. Chest 1974; 66: 647-651.

10. Chester EH, Belman JM, Bahler RC, Baum GL, Schey G, Buch P. Multidisciplinary treatment of chronic pulmonary insufficiency: the effect of physical training on cardiopulmonary performance in patients with chronic obstructive pulmonary disease. Chest 1977; 72: 695-702.

11. Mall RW, Meideros M. Objective evaluation of results of a pulmonary rehabilitation in a community hospital. Chest 1988; 94: 1156-1160.

12. Moser KM, Bokinsky GD, Savage RT, Archibold CJ, Hansen PR. Results of a comprehensive rehabilitation program. Arch Intern Med 1980; 140: 1596-1600.
13. Unger KM, Moser KM, Hansen P. Selection of an exercise program for patients with COPD. Heart Lung 1980; 9: 68-76.

14. Wasserman K, Whipp BJ, Koyal SN, Beaver WL. Anaerobic threshold and respiratory gas exchange during exercise. J Appl Physiol 1973; 35: 236-243.

15. Ahmaïdi S, Varray A, Savy Pacaux AM, Préfaut Ch. Cardiorespiratory fitness evaluation by the shuttle test in asthmatic subjects during aerobic training. Chest 1993; 103: 1135-1141.

16. Varray AL, Mercier JG, Terral CM, Préfaut Ch. Individualized aerobic and high intensity training for asthmatic children in an exercise readaptation program. Chest 1991; 99: 579-586.

17. Vallet G, Fontaine JL, Varray A, Préfaut Ch. Intérêt du réentraînement à l'effort individualisé au niveau du seuil ventilatoire, au cours de la bronchopneumopathie chronique obstructive de sévérité modérée. Rev Mal Respir 1994; 11: 493-501.

18. Vago P, Mercier J, Ramonatxo M, Préfaut Ch. Is ventilatory anaerobic threshold a good index of endurance capacity? Int J Sports Med 1987; 8(3): 190-195.

19. Préfaut Ch, Varray A, Vallet G. Pathophysiological basis of exercise training in patients with chronic obstructive lung disease. Eur Respir Rev 1995; 5(25): 51-58.

20. Pollock ML, Wilmore JH. Prescribing exercise for the apparently healthy. Guidelines and preliminary considerations. In: Pollock ML, Wilmore JH, eds. Exercise in Health and Disease. WB. Saunders Co., 2rd, 1990; 7: pp. 371-484.

21. Sadoul P, Polu JM. Invalidité respiratoire et son évaluation. Encycl Méd Chir Paris 1982; Poumon 6000 R10, 6.

22. American Thoracic Society. Evaluation of impairment/disability secondary to respiratory disorders. Am Rev Respir Dis 1986; 133: 1205-1209.

23. Quanjer H. Standardized lung function testing. Bull Eur Physiopathol Respir 1983; 19 (Suppl. 5): 1: 1-95.

24. Sue DY, Wasserman K, Moricca RB, Casaburi R. Metabolic acidosis during exercise in patients with chronic obstructive pulmonary disease. Chest 1988; 94: 931-938.

25. Massé-Biron J, Ahmaïdi S, Adam B, Préfaut Ch. Intérêt d'un réentraïnement aérobie individualisé au niveau du seuil ventilatoire chez des sujets âgés. Sci Sports 1993; 8: 251-259.

26. Bland JM, Altman DG. Statistical methods for assessing agreement between two methods of clinical measurement. Lancet 1986; i: 307-310.

27. Belman MJ, Kendregan BA. Exercise training fails to increase skeletal muscle enzymes in patients with chronic obstructive pulmonary disease. Am Rev Respir Dis 1991; 143: 905-912.

28. Begin P, Grassino A. Inspiratory muscle dysfunction and chronic hypercapnia in chronic obstructive pulmonary disease. J Appl Physiol 1991; 143: 905-912.

29. Kavannagh T, Yocoub MH, Mertens DJ, Kennedy J, Campbell RB, Sawyer P. Cardiorespiratory responses to exercise training after orthotopic cardiac transplantation. Circulation 1988; 77: 162-177.

30. Wanke TH, Formanek D, Lahrman H, Merkle M, Rausher $\mathrm{H}$, Zwick $\mathrm{H}$. Inspiratory muscle performance relative to the anaerobic threshold in patients with COPD. Eur Respir J 1993; 6: 1186-1191.

31. Gibbons ES, Jessup GT, Wells TD, Werthmann DA. Effect of various training intensity levels on anaerobic thresholds and aerobic capacity in females. J Sports Med 1983; 23: 315-318. 
32. Niederman MS, Clemente Ph, Fein AM, et al. Benefits of a multidisciplinary pulmonary rehabilitation program. Chest 1991; 99: 798-804.

33. Jakobsson P, Jordfeldt L, Brundin A. Skeletal muscle metabolites and fibre types in patients with advanced chronic obstructive pulmonary disease (COPD), with and without chronic respiratory failure. Eur Respir $J$ 1990; 3: 192-196.

34. Mohsenifar Z, Horak D, Brown V, Koerner K. Sensitive indices of improvement in a pulmonary rehabilitation program. Chest 1983; 83: 189-192.

35. Couser JI, Martinez FJ, Celli RB. Pulmonary rehabilitation that includes arm exercise reduces metabolic and ventilatory requirements for simple arm elevation. Chest 1993; 103: 37-41.

36. Corriveau M, Harris Ch, Chun DS, Keller C, Dolan GF. Relationship between multiple physiologic variables and change in exercise capacity after pulmonary rehabilitation program. J Cardiopulm Rehabil 1988; 8: 303-308.

37. Sergysels R, Degré S, Garcia-Herreros P, Willeput R, A de Coster. The ventilatory pattern during exercise in COPD. Bull Eur Physiopathol Respir 1979; 15: 57-70.

38. Fragoso CA, Clark TC, Kotch A. The tidal volume response to incremental exercise in COPD. Chest 1993; 103: 1438-1441.

39. Noseda A, Carpiaux J-P, Schmerber J, Valente F, Yernault J-C. Dyspnoea and flow volume curve during exercise in COPD patients. Eur Respir J 1994; 7: 279-285.

40. Babb TG, Viggiano B, Hurley B, Staats B, Rodarte JR. Effect of mild-to-moderate airflow limitation on exercise capacity. J Appl Physiol 1991; 70: 223-230.

41. Varray AL, Mercier JG, Savy-Pacaux AM, Préfaut Ch. Cardiac role in exercise limitation in asthmatic subjects with special reference to disease severity. Eur Respir J 1993; 6: 1011-1017. 